\title{
Infecções sexualmente transmissíveis em idosos: revisão integrativa da literatura
}

\author{
Sexually transmitted infections in the elderly: integrative literature review \\ Infecciones de transmisión sexual en los ancianos: revisión integradora de la literatura \\ Rosangela Jeniffer Soares Rosa ${ }^{1 *}$, Anne Eugênia Ledo Gonçalves Viana ${ }^{1}$, Luna Vitória Cajé Moura ${ }^{1}$, \\ Emilly Suzane Padilha da Silva ${ }^{1}$, Quésia de Almeida Dias ${ }^{1}$.
}

\section{RESUMO}

Objetivo: Compreender os fatores que vulnerabiliza a pessoa idosa concernente às Infecções Sexualmente Transmissíveis (IST), apresentar as repercussões físicas e psicológicas que acometem os idosos com ênfase na sexualidade e no diagnóstico de IST, a importância da educação em saúde desde a atenção básica, desse grupo populacional. Métodos: Revisão Integrativa da Literatura. Desenvolvida por meio das bases de dados Scientific Electronic Library Online (SCIELO), Biblioteca Virtual em Saúde (BVS) e Literatura Latino-Americana em Ciências da Saúde (LILACS) publicada entre o ano de 2016 a 2021 em língua portuguesa, selecionandose 20 artigos para análise e discussão. Resultados: Percebe-se que a disseminação de informação ao grupo de idosos faz-se muito necessária e são de extrema importância, ações que direcionam para educação em saúde adentrando na sexualidade para que obtenham segurança, direito ao prazer sexual. Considerações finais: Os idosos permanecem sexualmente ativos, porém existem informações insuficientes sobre prevenção das IST'S, contribuindo para o aumento da transmissão e contaminação. Os profissionais de saúde direcionam seus olhares aos mais jovens, colaborando para a falta de conhecimento da pessoa idosa.

Palavras-chave: IST, Pessoa idosa, Saúde do idoso, Educação em saúde, Sexualidade.

\begin{abstract}
Objective: Understand the factors that make the elderly vulnerable to Sexually Transmitted Infections (STIs), present the physical and psychological repercussions that affect the elderly with an emphasis on sexuality and the diagnosis of STIs, the importance of health education from primary care, of this group populational. Methods: Integrative Literature Review. Developed through the Scientific Electronic Library Online (SCIELO), Virtual Health Library (VHL) and Latin American Literature in Health Sciences (LILACS) databases, published between 2016 and 2021 in Portuguese, by selecting 20 articles for analysis and discussion. Results: It is noticed that the dissemination of information to the elderly group is very necessary and are extremely important, actions that direct health education into sexuality so that they obtain security, the right to sexual pleasure. Final considerations: The elderly remain sexually active, but there is insufficient information on the prevention of STI'S, contributing to the increase in transmission and contamination. Health professionals direct their gaze to younger people, contributing to the elderly person's lack of knowledge.
\end{abstract}

Keywords: IST, Elderly, Health of the elderly, Health education, Sexuality.

\section{RESUMEN}

Objetivo: Comprender los factores que hacen al adulto mayor vulnerable a las Infecciones de Transmisión Sexual (ITS), presentar las repercusiones físicas y psicológicas que afectan al adulto mayor con énfasis en la sexualidad y el diagnóstico de ITS, la importancia de la educación en salud desde la atención primaria, de este grupo poblacional. Métodos: Revisión Integrativa de la Literatura. Desarrollado a través de las bases de datos de la Biblioteca Científica Electrónica en Línea (SCIELO), Biblioteca Virtual en Salud (BVS) y Literatura Latinoamericana en Ciencias de la Salud (LILACS), publicadas entre 2016 y 2021 en portugués, mediante la selección de 20 artículos para análisis y discusión. Resultados: Se advierte que la difusión de información al colectivo de ancianos es muy necesaria y de suma importancia, acciones que orienten la educación en salud hacia la sexualidad para que obtengan seguridad, el derecho al placer sexual. Consideraciones finales: Los adultos mayores permanecen sexualmente activos, pero no hay información suficiente sobre la prevención de las ITS, lo que contribuye al aumento de la transmisión y la contaminación. Los profesionales de la salud dirigen su mirada hacia los más jóvenes, contribuyendo al desconocimiento del anciano.

Palabras clave: IST, Anciano, Salud de las personas mayors, Educación para la salud, Sexualidad.

${ }^{1}$ Faculdade Adventista da Bahia (FADBA), Cachoeira - BA. *E-mail: rosangela_jeniffer@hotmail.com 


\section{INTRODUÇÃO}

O número de idosos no Brasil é crescente, segundo dados do Instituto Brasileiro de Geografia e Estatística (IBGE) referente ao índice de envelhecimento resulta em cerca de $43,19 \%$ da população idosa no Brasil no ano de 2018, não obstante trazendo estatísticas futuras de $173,47 \%$ crescimento significativo desse grupo até 0 ano de 2060 (IBGE, 2018). Esses dados evidenciam a necessidade de adaptações para contemplar os idosos nas políticas sociais, previdenciárias, atendimentos sociais e, sobretudo na assistência à saúde da pessoa idosa (FERREIRA CO, et al., 2019).

A Organização Mundial de Saúde pontua que o programa brasileiro de combate à Síndrome da Imunodeficiência Adquirida (AIDS) é internacionalmente conhecido, através de ações que reduzem a transmissibilidade da doença, devido a medidas inovadoras de prevenção e da cobertura gratuita no tratamento, assegurada pelo Sistema Único de Saúde (SUS) (BRITO NM, et al., 2016).

O preconceito que permeia a sociedade afirmando que atividade sexual é exclusiva para o público de jovens com vitalidade, energia e fisicamente atraentes, demonstra que existe privação sexual composta de preconceitos, até mesmo partindo dos próprios idosos, da família, dos profissionais de saúde, interferindo no progresso da saúde sexual e no autocuidado com medidas de prevenção na terceira idade (BRITO NM, et al., 2016). A sexualidade é de direito humano, contribui para a plenitude em todas as fases da vida. Dessa maneira, a satisfação encontrada na prática sexual não desaparece na velhice (JÚNIOR PSS e MENDES PN., 2020).

A sexualidade dentro da realidade da pessoa idosa vem sendo estudada e tem sido evidenciado que os idosos permanecem com as suas atividades sexuais, contudo de forma diminuída de acordo com o processo fisiológico/ senescência, vale ressaltar que até após os 80 anos de idade muitos idosos sentem desejos e prazeres, assim, permanecem sexualmente ativos, todavia, muitas vezes de forma insegura por não considerarem vulneráveis a Infecções Sexualmente Transmissíveis(IST) (ANDRADE J, et al., 2017).

O envelhecimento é um processo fisiológico natural da vida denominado senescência, e as mudanças fazem parte e são importantes para o desenvolvimento, à vista disso, devido ao insuficiente conhecimento que a pessoa idosa possui sobre toda fisiologia do envelhecimento é comum haver a retração, devido ao surgimento de uma patologia ou por questões que envolvem cultura, abstendo-se da sua sexualidade (SANTOS MC, et al., 2017).

Torna-se então evidente que existem alterações comportamentais nos idosos pertinentes às práticas sexuais, devido ao crescimento da vitalidade e pelas descobertas de meios modernos que favorecem o desejo sexual, os estimulantes sexuais, como remédios, redes sociais e fantasias que facilitam a prática sexual. Informações sobre sexo seguro não estão sendo bem disseminadas ou voltadas ao público de pessoas idosas, de maneira que o número de infecções sexualmente transmissíveis nesse grupo vem pluralizando (ZANCO MR, et al., 2020). Um estudo realizado com idosos apontam que estes compreendem que a sexualidade está limitada à relação sexual, embora se saiba que vai muito além (VIEIRA KFL, et al., 2016).

Sendo assim, o planejamento de ações de saúde que incluam as necessidades sexuais dos idosos é de extrema importância, uma vez que o bem-estar e qualidade de vida também estão relacionados à sexualidade (SILVA AG, et al., 2017). A redução da amplitude das IST está diretamente relacionada ao conhecimento da população sobre estas infecções, principalmente sobre as medidas de prevenção ligadas à atenção primária (PINTO VM, et al., 2018).

Importante salientar que o tema é pertinente, com frequente discussão da temática, porém com evidente escassez em publicações, faz-se necessário que tais informações sobre a sexualidade da pessoa idosa seja disseminada com intuito de prevenir, quebrar tabus e proporcionar saúde sexual a esse público. Objetivou-se compreender os fatores que vulnerabilizam a contaminação da pessoa idosa, bem como entender as repercussões psicológicas e físicas do idoso acometido por IST e atuação dos profissionais da saúde atrelada a Unidade Básica de Saúde (UBS) no cuidado a sexualidade da pessoa idosa.

\section{MÉTODOS}

Trata-se de uma Revisão Integrativa da Literatura, as buscas dos artigos científicos foram realizadas nas bases de dados Scientific Electronic Library Online (SCIELO), Biblioteca Virtual em Saúde (BVS) e Literatura Latino-Americana em Ciências da Saúde (LILACS), foram utilizados sites e publicações institucionais do Ministério da Saúde e Organização Mundial de Saúde. 
Os descritores foram extraídos do Descritores em Ciência da Saúde (DeCS) sendo eles: IST, pessoa idosa, educação em saúde, sexualidade, saúde do idoso. Para a busca dos artigos os descritores mais utilizados foram sexualidade, pessoa idosa e IST. Na base SCIELO foi realizado o seguinte cruzamento: "sexualidade and educação em saúde" e foram encontrados 6 (seis) artigos para compor o estudo. Na BVS, houve o cruzamento dos descritores "pessoa idosa and IST and saúde do idoso", resultando em 9 (nove) artigos. No LILACS "pessoa idosa and IST" com 5 (cinco) artigos encontrados, que por fim resultou nos 20 artigos selecionados para compor o estudo. Foram selecionados apenas artigos científicos realizados no Brasil, em língua portuguesa, publicados no período de 2016 a maio de 2021, e aqueles disponíveis na íntegra para leitura (Figura 1).

Foram excluídos artigos científicos publicados em países estrangeiros, livros, resumos em língua estrangeira, os que foram publicados anteriores ao ano de 2016, que não correspondiam aos objetivos do trabalho e que apresentavam duplicidade. Os artigos eleitos foram submetidos a leitura completa e mais apurada para análise e extração de saberes a fim de contemplar esta pesquisa. Com base nos estudos analisados e selecionados como importante para elaboração da pesquisa foi registrado um número total de 268 artigos, 37 no LILACS, 158 no BVS e 73 na SCIELO (Figura 1).

Figura 1 - Fluxograma do processo de busca e identificação dos artigos

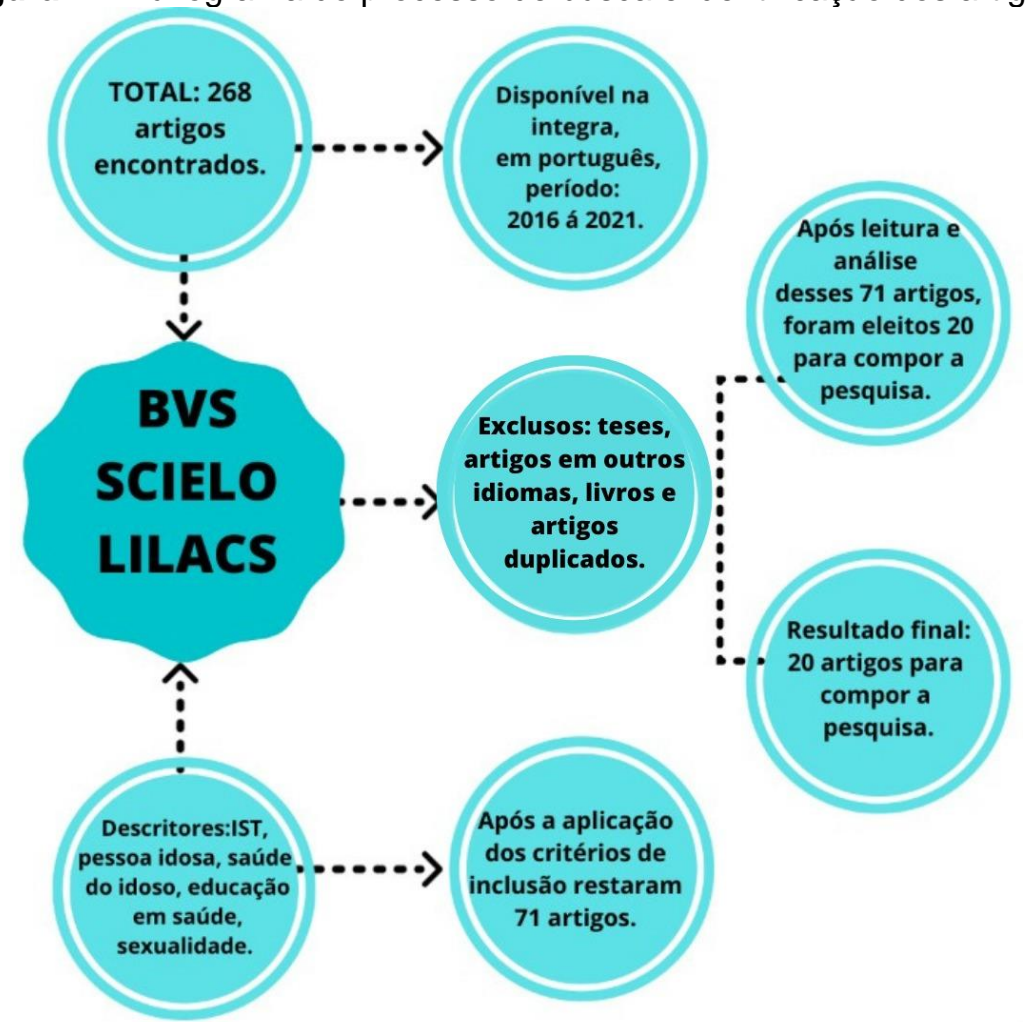

Fonte: Rosa RJS, et al., 2021.

Logo, foram selecionados 20 artigos, sendo 5 da base de dados LILACS, 9 BVS e 6 da SCIELO, sucedeu a exclusão de 248 artigos, 20 artigos foram eleitos e julgados como cruciais para este estudo, por seguinte foram selecionados 20 artigos para compor um quadro síntese, contendo os seguintes itens: autores, título do artigo, ano da publicação, local, nome da revista, tipo de estudo e resultados (Quadro 1).

\section{RESULTADOS}

Com base na realização da seleção das publicações, foram organizadas por autor, título do artigo, ano, revista, tipo de estudo, objetivo e resultados no Quadro 1. A partir da leitura dos artigos, emergiram três categorias: "Fatores relacionados à vulnerabilidade de pessoas idosas as IST'S", "Repercussões clínicas e psicológicas das IST'S nas pessoas idosas", "Educação em saúde na atenção básica acerca da sexualidade das pessoas idosas." 
Quadro 1- Síntese de artigos da amostra final, por ordem crescente. BVS, Scielo e LILACS

\begin{tabular}{|c|c|c|c|c|c|}
\hline Autor/Ano & Título do artigo & Revista & Tipo de estudo & Objetivo & Resultados \\
\hline $\begin{array}{l}\text { ALVES MA, et } \\
\text { al. (2017) }\end{array}$ & $\begin{array}{l}\text { As dificuldades } \\
\text { enfrentadas pelo } \\
\text { paciente idoso } \\
\text { diagnosticado com o } \\
\text { HIV: Olhar do } \\
\text { enfermeiro diante da } \\
\text { problemática. }\end{array}$ & $\begin{array}{c}\text { Revista Saúde } \\
\text { em Foco }\end{array}$ & $\begin{array}{l}\text { Revisão } \\
\text { sistemática de } \\
\text { literatura }\end{array}$ & $\begin{array}{l}\text { O trabalho objetivava compreender e } \\
\text { apontar os fatores que interferem de } \\
\text { forma significativa no diagnóstico, } \\
\text { adesão, aceitação, enfrentamento da } \\
\text { patologia, bem como contextualizar os } \\
\text { motivos que ainda fortalecem o } \\
\text { preconceito frente ao diagnóstico do } \\
\text { HIV em indivíduos idosos. }\end{array}$ & $\begin{array}{l}\text { O HIV/AIDS não está totalmente incorporado no processo } \\
\text { de saúde/doença do idoso, como outros adoecimentos } \\
\text { relacionados à idade, exemplos: Diabetes Mellitus e } \\
\text { Hipertensão Arterial. É necessário criar novos formatos } \\
\text { de educação em saúde cujo foco central seja o alcance } \\
\text { de indivíduos descritos. Colocar a disposição à realização } \\
\text { de testes rápidos, grupos de orientação sexual, pois } \\
\text { quanto mais cedo for realizado o diagnóstico, mais cedo } \\
\text { se começa o tratamento, diminuindo assim a taxa de co- } \\
\text { morbidades e morbimortalidade relacionadas ao HIV. }\end{array}$ \\
\hline $\begin{array}{l}\text { AMARAL SV, } \\
\text { et al. (2020) }\end{array}$ & $\begin{array}{l}\text { Conhecimento e } \\
\text { comportamento de um } \\
\text { grupo de idosos frente } \\
\text { às infecções } \\
\text { sexualmente } \\
\text { transmissíveis. }\end{array}$ & $\begin{array}{l}\text { Revista } \\
\text { Eletrônica } \\
\text { Acervo Saúde }\end{array}$ & $\begin{array}{l}\text { Pesquisa de } \\
\text { campo, de } \\
\text { delineamento } \\
\text { quantitativo }\end{array}$ & $\begin{array}{l}\text { Compreender sobre o comportamento } \\
\text { e o conhecimento de um grupo de } \\
\text { idosos frente às Infecções } \\
\text { Sexualmente Transmissíveis (IST's). }\end{array}$ & $\begin{array}{l}\text { O estudo evidenciou que os idosos possuem certo } \\
\text { conhecimento acerca das IST'S e de suas formas de } \\
\text { transmissão. Dentre as IST'S a mais conhecida pelos } \\
\text { entrevistados é a HIV/AIDS ( } 64 \%) \text {. Porém a maioria adota } \\
\text { comportamento de risco ao não usar o preservativo nas } \\
\text { relações sexuais }(76,3 \%) \text { e } 81,4 \% \text { deles não se } \\
\text { consideram vulneráveis para adquirir uma IST, } \\
\text { justificando tal fato por terem parceiro fixo. Um achado } \\
\text { positivo é a ciência da existência da vacina contra a } \\
\text { hepatite B (50,8\%), identificando-a como uma doença } \\
\text { imunoprevenível, disponível inclusive para sua faixa } \\
\text { etária }\end{array}$ \\
\hline $\begin{array}{l}\text { ANDRADE J, } \\
\text { et al. (2017) }\end{array}$ & $\begin{array}{l}\text { Vulnerabilidade de } \\
\text { idosos a infecções } \\
\text { sexualmente } \\
\text { transmissíveis. }\end{array}$ & $\begin{array}{l}\text { Acta Paulista } \\
\quad \text { de } \\
\text { Enfermagem }\end{array}$ & $\begin{array}{c}\text { Estudo } \\
\text { transversal }\end{array}$ & $\begin{array}{l}\text { Identificar a prevalência e fatores } \\
\text { associados às Infecções Sexualmente } \\
\text { Transmissíveis (IST) em idosos. }\end{array}$ & $\begin{array}{l}\text { Os resultados mostram a vulnerabilidade dos idosos às } \\
\text { IST. Sugerem-se estratégias que favoreçam a educação } \\
\text { permanente dos profissionais na temática. }\end{array}$ \\
\hline $\begin{array}{l}\text { ARAÚJO JI, } \\
\text { ANDRADE FG } \\
(2017)\end{array}$ & $\begin{array}{l}\text { Prolongamento da } \\
\text { vida sexual entre a } \\
\text { população idosa: } \\
\text { Conquistas e desafios. }\end{array}$ & $\begin{array}{c}\text { Congresso } \\
\text { internacional } \\
\text { envelheciment } \\
\text { o humano }\end{array}$ & $\begin{array}{c}\text { Revisão } \\
\text { sistemática de } \\
\text { literatura } \\
\text { científica }\end{array}$ & $\begin{array}{l}\text { Este estudo objetiva expor a } \\
\text { necessidade de um processo de } \\
\text { preparação por parte dos profissionais } \\
\text { de saúde, para que possam } \\
\text { desenvolver estratégias específicas } \\
\text { acerca da realidade dos idosos e da } \\
\text { naturalidade sexual desses indivíduos. }\end{array}$ & $\begin{array}{l}\text { A sexualidade na terceira idade ainda é caracterizada por } \\
\text { conceitos errôneos por parte dos próprios idosos, } \\
\text { familiares e pelos profissionais de saúde. Concluiu-se } \\
\text { que com o aumento da população idosa, também houve } \\
\text { um crescimento significativo das IST, evidenciando que } \\
\text { muito se fez a fim de proporcionar facilidades para o } \\
\text { prolongamento sexual, no entanto, pouco foi feito em } \\
\text { informação preventiva direcionada às pessoas idosas. }\end{array}$ \\
\hline
\end{tabular}




\begin{tabular}{|c|c|c|c|c|c|}
\hline Autor/Ano & Título do artigo & Revista & Tipo de estudo & Objetivo & Resultados \\
\hline $\begin{array}{l}\text { BEZERRA LL, } \\
\text { et al (2017) }\end{array}$ & $\begin{array}{l}\text { Abordagem das ist por } \\
\text { enfermeiro (as): } \\
\text { revisão integrativa de } \\
\text { literatura }\end{array}$ & $\begin{array}{c}\text { II } \\
\text { CONBRACIS }\end{array}$ & $\begin{array}{l}\text { Revisão } \\
\text { Integrativa de } \\
\text { Literatura }\end{array}$ & $\begin{array}{l}\text { Analisar como é realizada, na prática, a } \\
\text { abordagem dos enfermeiros (as) frente } \\
\text { às pessoas que possuem alguma IST. }\end{array}$ & $\begin{array}{l}\text { Observou-se que as principais formas de prevenção } \\
\text { contra IST é através do aconselhamento, ações voltadas } \\
\text { à promoção de saúde e o uso do preservativo em todas } \\
\text { as relações sexuais. A educação em saúde compóe } \\
\text { invariavelmente o cuidado de enfermagem, tanto no nível } \\
\text { individual, quanto coletivo na atenção as IST/Aids. } \\
\text { Constatou-se que são necessárias estratégias que } \\
\text { atendam as particularidades dos usuários e de grupos } \\
\text { específicos, como exemplo, adolescentes, idosos e } \\
\text { pessoas com deficiência. O diagnóstico pode ser feito a } \\
\text { partir de uma avaliação clínica em conjunto com testes e } \\
\text { exames complementares; o tratamento é realizado } \\
\text { através da prescrição de medicamentos específicos para } \\
\text { as patologias identificadas. Os enfermeiros (as) } \\
\text { encontram diversas barreiras que dificultam a assistência } \\
\text { qualificada, como falta de matérias e infraestrutura } \\
\text { inadequada }\end{array}$ \\
\hline $\begin{array}{l}\text { BRITO NM, et } \\
\text { al. (2016) }\end{array}$ & $\begin{array}{l}\text { Idosos, infecções } \\
\text { sexualmente } \\
\text { transmissíveis e AIDS: } \\
\text { conhecimentos e } \\
\text { percepção de risco. }\end{array}$ & $\begin{array}{l}\text { ABCS Health } \\
\text { Sciences }\end{array}$ & $\begin{array}{l}\text { Estudo } \\
\text { descritivo de } \\
\text { natureza } \\
\text { quantitativa }\end{array}$ & $\begin{array}{l}\text { Investigar o conhecimento e verificar a } \\
\text { percepção de risco de idosos quanto à } \\
\text { contaminação por Infecções } \\
\text { Sexualmente Transmissíveis (IST) e } \\
\text { HIV. }\end{array}$ & $\begin{array}{l}\text { O estudo descritivo com os grupos de idosos, mostra que } \\
\text { essa população se considera pouco vulnerável à } \\
\text { contaminação ou não se percebe em risco, o que os torna } \\
\text { susceptíveis ao perigo da infecção, favorecendo o } \\
\text { aumento do índice de idosos infectados no cenário } \\
\text { nacional. }\end{array}$ \\
\hline $\begin{array}{l}\text { CASSÉTE JB, } \\
\text { et al. (2016) }\end{array}$ & $\begin{array}{l}\text { HIV/aids em idosos: } \\
\text { estigmas, trabalho e } \\
\text { formação em saúde. }\end{array}$ & $\begin{array}{l}\text { Revista } \\
\text { Brasileira de } \\
\text { Geriatria e } \\
\text { Gerontologia }\end{array}$ & $\begin{array}{l}\text { Pesquisa } \\
\text { qualitativa, de } \\
\text { caráter } \\
\text { exploratório }\end{array}$ & $\begin{array}{l}\text { Buscar analisar a atuação de } \\
\text { profissionais de saúde em idosos com } \\
\text { diagnóstico de HIV/aids em um serviço } \\
\text { público de saúde em um município de } \\
\text { porte médio de Minas Gerais. }\end{array}$ & $\begin{array}{l}\text { Pela análises, conclui-se que os estigmas vinculados à } \\
\text { sexualidade da pessoa idosa estão presentes no } \\
\text { trabalho dos profissionais entrevistados,e interferem nos } \\
\text { processos de saúde e adoecimento. }\end{array}$ \\
\hline $\begin{array}{l}\text { FERREIRA } \\
\text { CO, et al. } \\
\text { (2019) }\end{array}$ & $\begin{array}{l}\text { Vulnerabilidade a } \\
\text { infecções sexualmente } \\
\text { transmissíveis em } \\
\text { idosos usuários de um } \\
\text { centro de testagem e } \\
\text { aconselhamento }\end{array}$ & $\begin{array}{l}\text { Ciências da } \\
\text { Saúde } \\
\text { UNIPAR }\end{array}$ & $\begin{array}{l}\text { Estudo analítico } \\
\text { observacional } \\
\text { transversal, } \\
\text { exploratório }\end{array}$ & $\begin{array}{l}\text { Investigar situações de vulnerabilidade } \\
\text { relacionadas a IST em idosos usuários } \\
\text { de um Centro de Testagem e } \\
\text { Aconselhamento para DST/Aids de um } \\
\text { município de médio porte do estado da } \\
\text { Bahia, Brasil, no período de } 2006 \text { a } \\
2012 \text {. Foram utilizados dados } \\
\text { secundários de } 233 \text { usuários, com } 60 \\
\text { anos ou mais, coletados dos } \\
\text { Formulários de Entrada do Sistema de } \\
\text { Informação do CTA, prontuários } \\
\text { clínicos e folha de descrição do } \\
\text { atendimento. }\end{array}$ & $\begin{array}{l}\text { Os resultados evidenciaram práticas sexuais inseguras e } \\
\text { elevada vulnerabilidade dos idosos às IST, havendo } \\
\text { necessidade de ações preventivas direcionadas a esse } \\
\text { grupo populacional, considerando suas necessidades e } \\
\text { especificidades. }\end{array}$ \\
\hline
\end{tabular}




\begin{tabular}{|c|c|c|c|c|c|}
\hline Autor/Ano & Título do artigo & Revista & Tipo de estudo & Objetivo & Resultados \\
\hline $\begin{array}{l}\text { JÚNIOR PSS, } \\
\text { MENDES PN } \\
\text { (2020). }\end{array}$ & $\begin{array}{l}\text { Sexualidade do idoso: } \\
\text { intervenções do } \\
\text { enfermeiro para a } \\
\text { prevenção das } \\
\text { infecções } \\
\text { sexualmente } \\
\text { transmissíveis. }\end{array}$ & $\begin{array}{l}\text { Pesquisa, } \\
\text { Sociedade e } \\
\text { Desenvolvime } \\
\text { nto. }\end{array}$ & $\begin{array}{c}\text { Revisão } \\
\text { bibliográfica }\end{array}$ & $\begin{array}{l}\text { Descrever as intervenções de } \\
\text { enfermagem para a prevenção das } \\
\text { infecções } \\
\text { sexualmente transmissíveis na } \\
\text { terceira idade. }\end{array}$ & $\begin{array}{l}\text { O enfermeiro pode atuar de diversas maneiras na } \\
\text { prevenção de IST em idosos, uma delas é a palestra } \\
\text { e rodas de conversas com idosos, visto que } \\
\text { atualmente o jovem tem mais acesso à prevenção de } \\
\text { IST do que idosos. No Brasil, infelizmente, ainda } \\
\text { existe esse "preconceito" em razão da sexualidade } \\
\text { dos idosos e que eles não podem ter doenças } \\
\text { sexualmente transmissíveis pois não vivem esse } \\
\text { importante aspecto da vida. }\end{array}$ \\
\hline $\begin{array}{l}\text { LIMA JS, et al. } \\
\qquad(2021)\end{array}$ & $\begin{array}{l}\text { O conhecimento dos } \\
\text { idosos acerca das } \\
\text { infecções sexualmente } \\
\text { transmissíveis. }\end{array}$ & $\begin{array}{l}\text { Caderno de } \\
\text { Graduação- } \\
\text { Ciências } \\
\text { Biológicas e } \\
\text { da Saúde- } \\
\text { UNIT- } \\
\text { ALAGOAS }\end{array}$ & $\begin{array}{l}\text { Revisão } \\
\text { integrativa de } \\
\text { literatura }\end{array}$ & $\begin{array}{l}\text { Este estudo tem como objetivo } \\
\text { identificar o conhecimento dos idosos } \\
\text { acerca das doenças sexualmente } \\
\text { transmissíveis e a adoção de medidas } \\
\text { preventivas pelos mesmos. }\end{array}$ & $\begin{array}{l}\text { A literatura ressalta que muitos idosos não possuem total } \\
\text { conhecimento das ISTs devido ao tabu estabelecido } \\
\text { neste assunto. Sendo assim, é importante que o } \\
\text { profissional de enfermagem possa implementar ações } \\
\text { específicas e contínuas voltadas à saúde sexual do idoso. }\end{array}$ \\
\hline $\begin{array}{l}\text { MONTE CF, et } \\
\text { al. }(2021)\end{array}$ & $\begin{array}{l}\text { Idosos frente a } \\
\text { infecções sexualmente } \\
\text { transmissíveis: uma } \\
\text { revisão integrativa }\end{array}$ & $\begin{array}{l}\text { Brazilian } \\
\text { Journal of } \\
\text { Health Review }\end{array}$ & $\begin{array}{l}\text { Revisão } \\
\text { integrativa }\end{array}$ & $\begin{array}{l}\text { O objetivo do estudo foi levantar } \\
\text { informações sobre o IST em idosos, } \\
\text { evidenciando dois eixos temáticos, o } \\
\text { conhecimento sobre a temática e os } \\
\text { fatores de risco desse grupo }\end{array}$ & $\begin{array}{l}\text { Conclui-se que se faz necessário campanhas e } \\
\text { realização de medidas e estratégias preventivas voltadas } \\
\text { para esse público, a fim de diminuir a quantidade de } \\
\text { casos em idosos. }\end{array}$ \\
\hline $\begin{array}{l}\text { PINTO VM, et } \\
\text { al. (2018) }\end{array}$ & $\begin{array}{c}\text { Fatores associados às } \\
\text { infecções sexualmente } \\
\text { transmissíveis: } \\
\text { inquérito populacional } \\
\text { no município de São } \\
\text { Paulo, Brasil }\end{array}$ & $\begin{array}{l}\text { Ciência \& } \\
\text { Saúde } \\
\text { Coletiva }\end{array}$ & $\begin{array}{l}\text { Estudo de corte } \\
\text { transversal, com } \\
\text { inquérito } \\
\text { populacional }\end{array}$ & $\begin{array}{l}\text { Descrevemos a frequência de } \\
\text { infecções sexualmente transmissíveis } \\
\text { (IST), os fatores associados e as } \\
\text { orientações recebidas dos profissionais } \\
\text { de saúde entre homens e mulheres no } \\
\text { município de São Paulo. }\end{array}$ & $\begin{array}{l}\text { A elevada proporção de antecedentes de IST entre a } \\
\text { população do município e os resultados deste estudo } \\
\text { possibilitaram a construção, implementação e avaliação } \\
\text { de políticas públicas de saúde para o enfrentamento das } \\
\text { IST incluindo o HIV, com diminuição de barreiras de } \\
\text { acesso aos preservativos e criação de um app para } \\
\text { prevenção. }\end{array}$ \\
\hline $\begin{array}{l}\text { RODRIGUES } \\
\text { MS, et al. } \\
\text { (2019) }\end{array}$ & $\begin{array}{l}\text { Obstáculos } \\
\text { enfrentados pela } \\
\text { Enfermagem na } \\
\text { prevenção de } \\
\text { infecções sexualmente } \\
\text { transmissíveis na } \\
\text { terceira idade. }\end{array}$ & $\begin{array}{l}\text { Revista } \\
\text { Eletrônica } \\
\text { Acervo Saúde }\end{array}$ & $\begin{array}{l}\text { Revisão } \\
\text { integrativa com } \\
\text { abordagem } \\
\text { qualitativa }\end{array}$ & $\begin{array}{l}\text { Identificar os obstáculos enfrentados } \\
\text { pela Enfermagem na prevenção de } \\
\text { infecções sexualmente transmissíveis } \\
\text { na terceira idade. }\end{array}$ & $\begin{array}{l}\text { A perpetuação de padrões arcaicos sobre a sexualidade, } \\
\text { falta de conhecimento dos idosos, falhas nas ações } \\
\text { educativas da equipe de Enfermagem e a resistência do } \\
\text { sujeito idoso quanto ao uso do preservativo foram alguns } \\
\text { dos obstáculos encontrados. }\end{array}$ \\
\hline $\begin{array}{l}\text { SANTOS FM, } \\
\text { et al. (2020) }\end{array}$ & $\begin{array}{l}\text { Idoso e HIV: um } \\
\text { desafio para o } \\
\text { enfermeiro nas } \\
\text { estratégias de } \\
\text { prevenção }\end{array}$ & $\begin{array}{l}\text { BIUS-Boletim } \\
\text { Informativo } \\
\text { Unimotrisaúde } \\
\text { em } \\
\text { Sociogerontolo } \\
\text { gia }\end{array}$ & $\begin{array}{l}\text { Pesquisa de } \\
\text { revisão } \\
\text { integrativa da } \\
\text { literatura }\end{array}$ & $\begin{array}{l}\text { Descrever o desafio para o enfermeiro } \\
\text { nas estratégias de prevenção do HIV } \\
\text { em idoso. }\end{array}$ & $\begin{array}{l}\text { Os idosos mantêm a vida sexual ativa e estão expostos } \\
\text { às infecções sexualmente transmissíveis, em especial ao } \\
\text { vírus da imunodeficiência humana (HIV), a prática sexual } \\
\text { não aumenta a vulnerabilidade dos idosos em relação à } \\
\text { infecção pelo HIV, e sim a prática sexual desprotegida, } \\
\text { fato que é atribuído a todas as idades e não apenas aos } \\
\text { idosos. }\end{array}$ \\
\hline
\end{tabular}




\begin{tabular}{|c|c|c|c|c|c|}
\hline Autor/Ano & Título do artigo & Revista & Tipo de estudo & Objetivo & Resultados \\
\hline $\begin{array}{c}\text { SANTOS MC, } \\
\text { et al. (2017) }\end{array}$ & $\begin{array}{c}\text { Percepções e } \\
\text { vivências de idosos } \\
\text { sobre sua sexualidade }\end{array}$ & $\begin{array}{l}\text { Almanaque } \\
\text { multidisciplinar } \\
\text { de pesquisa }\end{array}$ & $\begin{array}{l}\text { Pesquisa } \\
\text { qualitativa com } \\
\text { abordagem } \\
\text { descritiva }\end{array}$ & $\begin{array}{l}\text { O presente estudo teve como objetivo } \\
\text { conhecer as percepções e vivências de } \\
\text { idosos assistidos em um centro de } \\
\text { referência para pessoas idosas. }\end{array}$ & $\begin{array}{l}\text { A sexualidade é um fator importante para que os idosos } \\
\text { vivam melhor, contudo é necessário conhecer como eles } \\
\text { a percebem e a vivenciam, pois tal conhecimento é de } \\
\text { suma relevância para subsidiar os profissionais de saúde, } \\
\text { bem como para planejar políticas públicas voltadas para } \\
\text { essas pessoas. }\end{array}$ \\
\hline $\begin{array}{l}\text { SANTOS MC, } \\
\text { et al. (2018) }\end{array}$ & $\begin{array}{l}\text { Diagnósticos de } \\
\text { enfermagem para } \\
\text { mulheres idosas com } \\
\text { vulnerabilidade ao } \\
\text { HIV/aids }\end{array}$ & $\begin{array}{l}\text { Revista } \\
\text { brasileira de } \\
\text { enfermagem } \\
\text { REBEn }\end{array}$ & $\begin{array}{l}\text { Estudo de } \\
\text { natureza } \\
\text { exploratória } \\
\text { descritiva, }\end{array}$ & $\begin{array}{l}\text { Classificar os diagnósticos no quadro } \\
\text { conceitual de vulnerabilidade de Ayres } \\
\text { e na teoria do autocuidado de Orem; } \\
\text { elaborar definições operacionais de } \\
\text { diagnósticos de enfermagem para } \\
\text { mulheres idosas com vulnerabilidade } \\
\text { ao HIV/aids. }\end{array}$ & $\begin{array}{l}\text { Foram classificados } 70 \text { diagnósticos de enfermagem no } \\
\text { quadro conceitual de vulnerabilidade de Ayres e na teoria } \\
\text { do autocuidado de Orem, e foram construídas as suas } \\
\text { definições operacionais, em que } 75,7 \% \text { destas foram } \\
\text { validadas. }\end{array}$ \\
\hline $\begin{array}{l}\text { SILVA AG, et } \\
\text { al. (2018) }\end{array}$ & $\begin{array}{l}\text { Revisão integrativa da } \\
\text { literatura: assistência } \\
\text { de enfermagem à } \\
\text { pessoa idosa com HIV }\end{array}$ & $\begin{array}{l}\text { Rev Bras } \\
\text { Enferm }\end{array}$ & $\begin{array}{l}\text { Revisão } \\
\text { integrativa da } \\
\text { literatura }\end{array}$ & $\begin{array}{l}\text { Identificar na literatura } \begin{array}{c}\text { brasileira as } \\
\text { evidências científicas } \\
\text { sobre a }\end{array} \\
\text { assistência de enfermagem ao idoso } \\
\text { portador do HIV. }\end{array}$ & $\begin{array}{l}\text { Os estudos abordam a assistência de enfermagem ainda } \\
\text { através de uma clínica baseada nos diagnósticos da } \\
\text { NANDA com forte abordagem individualizante e baixa } \\
\text { consideração dos aspectos sociais }\end{array}$ \\
\hline $\begin{array}{l}\text { THEIS LC, } \\
\text { GOUVÊA DL, } \\
\text { (2019). }\end{array}$ & $\begin{array}{l}\text { Percepção dos Idosos } \\
\text { em Relação a Vida } \\
\text { Sexual e as Infecções } \\
\text { Sexualmente } \\
\text { Transmissíveis na } \\
\text { Terceira Idade. }\end{array}$ & $\begin{array}{l}\text { Rev. bras. } \\
\text { ciênc. saúde }\end{array}$ & $\begin{array}{l}\text { Estudo } \\
\text { qualitativo com } \\
\text { abordagem } \\
\text { descritiva, }\end{array}$ & $\begin{array}{l}\text { Conhecer a percepção dos idosos em } \\
\text { relação à vida sexual na terceira idade } \\
\text { e às infecções sexualmente } \\
\text { transmissíveis }\end{array}$ & $\begin{array}{l}\text { A partir da análise das falas dos entrevistados, } \\
\text { emergiram três categorias de análise, sendo elas: } \\
\text { sexualidade na terceira idade: os aspectos físicos e } \\
\text { emocionais sob o olhar dos idosos; a percepção dos } \\
\text { idosos sobre as infecções sexualmente transmissíveis; a } \\
\text { visão do idoso e o seu entendimento sobre a prevenção } \\
\text { de infecções sexualmente transmissíveis }\end{array}$ \\
\hline $\begin{array}{l}\text { VIEIRA KF, et } \\
\text { al. (2016) }\end{array}$ & $\begin{array}{l}\text { A sexualidade na } \\
\text { velhice: } \\
\text { representações sociais } \\
\text { de idosos } \\
\text { frequentadores de um } \\
\text { grupo de convivência. }\end{array}$ & $\begin{array}{l}\text { Psicologia: } \\
\text { ciência e } \\
\text { profissão }\end{array}$ & $\begin{array}{l}\text { Pesquisa } \\
\text { descritiva com } \\
\text { abordagem } \\
\text { qualitativa }\end{array}$ & 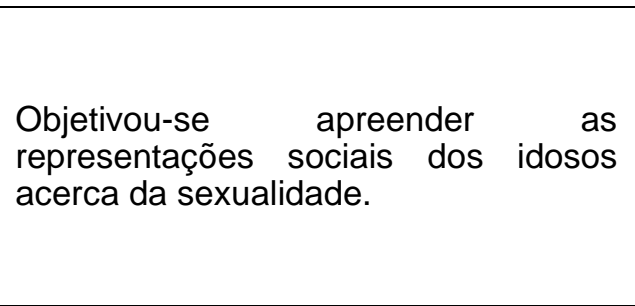 & $\begin{array}{l}\text { Observou-se que as representações sociais da } \\
\text { sexualidade se apresentaram de forma semelhante à } \\
\text { descrição científica, demonstrando similaridades entre o } \\
\text { senso comum e o conhecimento erudito. Acredita-se que } \\
\text { este estudo possa contribuir de forma parcimoniosa para } \\
\text { a promoção de mudanças de atitudes acerca da } \\
\text { sexualidade da pessoa idosa, trazendo benefícios para a } \\
\text { qualidade de vida dessa população. }\end{array}$ \\
\hline $\begin{array}{c}\text { ZANCO MR, } \\
\text { et al (2020) }\end{array}$ & $\begin{array}{l}\text { Sexualidade da } \\
\text { pessoa idosa: } \\
\text { principais desafios } \\
\text { para a atuação do } \\
\text { enfermeiro na atenção } \\
\text { primária em saúde }\end{array}$ & $\begin{array}{l}\text { Brazilian } \\
\text { journal of } \\
\text { health review }\end{array}$ & $\begin{array}{l}\text { Pesquisa } \\
\text { exploratória- } \\
\text { descritiva }\end{array}$ & $\begin{array}{l}\text { Descrever os principais desafios para o } \\
\text { enfermeiro da Atenção Primária em } \\
\text { Saúde atuar sobre a sexualidade da } \\
\text { pessoa idosa. }\end{array}$ & $\begin{array}{l}\text { Ao término desse trabalho verificamos que há } \\
\text { necessidade de novas propostas de trabalho, como a } \\
\text { necessidade de novas pesquisas sobre o assunto } \\
\text { englobando todas as transformações no envelhecer. }\end{array}$ \\
\hline
\end{tabular}

Fonte: Rosa RJS, et al., 2021. 


\section{Fatores relacionados à vulnerabilidade da pessoa idosas a Infecções Sexualmente Transmissíveis}

O atual contexto cultural abarrotado de restrições, preconceitos e o equívoco que desvincula os idosos do deleite das práticas sexuais devido ao avanço da idade, decréscimo da atividade e energia sexual, sucede a dificuldade de a pessoa idosa compreender que também podem ser vulneráveis as IST's, sem devidas informações voltadas para esse grupo, consequentemente temos elevação do número de casos diagnosticados (THEIS LC e GOUVÊA DL, 2019; BRITO NM, et al., 2016; ANDRADE J, et al., 2017).

As estatísticas referentes às IST na terceira idade tem ampliado significativamente em nível mundial, o aumento de diagnósticos junto a notificações revela dados alarmantes (MONTE CF, et al., 2021). Sendo estes números camuflados pela falta de informação, preconceito e tabus culturais. As IST estão em todas as classes sociais e idades, mas, os números de casos de AIDS na terceira idade no Brasil cresceu como em nenhuma outra faixa etária, ultrapassando o número de casos entre adolescentes de 15 a 19 anos (THEIS LC e GOUVÊA DL., 2019).

Há escassez de informações sobre a sexualidade voltada à pessoa idosa. O avanço da tecnologia para desempenho sexual, a discriminação da mulher idosa, a precariedade financeira, a dificuldade que possuem para diligenciar a relação sexual segura, a ausência de base acadêmica e escolaridade baixa, tais fatores contribuem para a vulnerabilidade principalmente de mulheres idosas à contaminação por algumas IST (SANTOS FM, et al., 2020).

Um estudo realizado por Theis LC e Gouvêa DL (2019), seguiu com entrevista de dez idosos, cinco do sexo masculino e cinco do sexo feminino com a idade entre 66 a 77 anos, destes mais da metade $60 \%$ pontuaram manter uma vida sexual ativa. Dentro disso, percebe-se que os idosos possuem atividade sexual de forma regular de acordo com suas condições fisiológicas e que os tabus proporcionam a vulnerabilidade às IST 'S nesse grupo, as mesmas poderiam ser evitadas através da prevenção e do acompanhamento na rede primária. Pesquisa realizada no Brasil, na região sul, sobre sexualidade apontou que as informações relacionadas à saúde sexual e IST são buscadas nos meios de comunicação, na internet, e que os participantes não relataram diálogo com os profissionais de saúde sobre sexualidade em suas consultas (ANDRADE J, et al., 2017).

Outra pesquisa pontuou que as pessoas idosas estão propensas a adquirirem algum tipo de IST em razão da falta de informação sobre preservativo como forma de prevenção, e que o nível de escolaridade também contribui para contaminação desse grupo, devido ao conhecimento insuficiente que a pessoa idosa possui associa-se ao receio, constrangimento, à falta de entendimento de como manusear o preservativo, conceitos equivocados de utilidade somente como barreira contra a gravidez, assim a resistência do uso do preservativo aumenta os novos casos de IST's neste grupo, ademais, atualmente cresceu o aumento de idosos fazendo uso de remédios para ereção, assim aumentando o número de relações sexuais desprotegidas gerando contaminação (BRITO NM, et al., 2016).

De acordo com o estudo de Andrade J, et al. (2017) os idosos que participaram da pesquisa e positivaram para algum tipo de IST's negaram fazer uso de preservativo, apontando para um dos maiores aspectos de vulnerabilidade. Dessa forma, um dos estudos destaca que um grupo de pessoas idosas com predominância do sexo feminino atendidas na rede primária de Campinas não utilizavam preservativos, e vale destacar que mais da metade desse grupo, $81 \%$, nunca utilizaram preservativo, as mesmas acreditavam que, a não existência de rotação de parceiros evita os riscos de contaminação (SANTOS FM, et al., 2010).

Sobre a percepção de risco que os idosos possuíam com relação a adquirir infecções sexualmente transmissíveis, $74,4 \%$ responderam que não havia possibilidades de contrair alguma IST ou/e HIV, 16,4\% acreditavam ter riscos insignificantes de contaminação. As justificativas eram baseadas na ausência de atividade sexual e parceiro fixo (BRITO NM, et al., 2016).

Em um estudo realizado na região Sul do país foi identificada a existência de algumas lacunas em relação ao conhecimento dos idosos a respeito do HIV/aids, como formas de transmissão, conceitos, as vulnerabilidades às quais estão expostos, e de outros fatores que contribuem para a aquisição da doença 
nessa faixa etária. Assim, é possível perceber que a disseminação de informação a esse grupo se faz muito necessária, e são de suma importância ações direcionadas à educação em saúde adentrando na sexualidade para que obtenham segurança, direito ao prazer sexual e conhecimento sobre suas vulnerabilidades (BRITO NM, et al., 2016).

Portanto, entende-se ser desafiador a concretização do cuidado qualificado quanto à prevenção dos idosos, pois depende do comprometimento dos profissionais, e estes precisam estar capacitados e motivados para trabalhar os aspectos da saúde na pessoa idosa (SANTOS FM, et al, 2020). O profissional por não reconhecer, entender a sexualidade na terceira idade pode confundir os sinais e sintomas de IST com os fatores normais do processo de senescência (ARAÚJO JI e ANDRADE FG, 2017).

Com a carência de projetos, falta de ações educativas que deveriam ser realizadas pelos profissionais da saúde, a prestação de serviço do enfermeiro à pessoa idosa se torna deficiente, principalmente quando se trata de sexualidade. A saúde sexual da pessoa idosa é subestimada, carregada de preconceitos e ignorância, sendo negligenciada até nos pontos de saúde pelos profissionais (VIEIRA KF, et al., 2016; ZANCO MR, et al., 2020).

\section{Repercussões clínicas e psicológicas das IST'S nas pessoas idosas}

Sabe-se que as repercussões das IST's são negativas e afetam tanto a saúde física quanto a mental, provocando um conjunto de desregulações no indivíduo idoso e ocasionando o declínio: cotidiano, psicológico, social, financeiro e em relações familiares. Estudos apontam que familiares se surpreendem com o diagnóstico por crer que seria impossível a transmissão do vírus por via sexual neste contexto etário (CASSÉTTE JB, et al., 2016,).

O estudo de Theis LC e Gouvêa DL (2019) evidenciou que algumas pessoas idosas se importam com o uso de preservativo devido ao medo da contaminação, e reconhecem as condições que vivem quanto ao processo de senescência, a queda da imunidade e possuem consciência do processo de tratamento, apresentando também o medo de ficar sozinho e a perda da alegria, entretanto, isso não se repete em tantos estudos.

Pacientes com diagnósticos de HIV/AIDS são percebidos pelos profissionais de saúde expressando de forma nítida o medo, insegurança, desesperança pertinente ao convívio com a miserável doença que gera tristeza e desolação; com a impossibilidade de reversão; estigmas e preconceitos relacionados ao HIV/AIDS que caracterizam a doença como "formas" ilícitas de viver, resultando em exclusão social e muita desolação desses pacientes. Contudo, acredita-se ser provável que para além das sequelas emocionais e físicas, as questões sexuais tornam-se evidentes provocando descontentamento e desânimo frente a prática sexual (CASSÉTTE JB, et al., 2016).

O aumento do número de casos diagnosticados deveria causar inquietação em diversos setores, como por exemplo, nos profissionais de saúde, nos conselheiros de saúde, governo e no país frente a um resultado de problemas físicos, adoecimento psicológico e exclusão social. De acordo com Cassétte JB, et al (2016) existem vários impactos que norteiam a pessoa idosa diagnosticada com alguma IST's, os profissionais de saúde apontam que entre eles estão: a tristeza, não aceitação do diagnóstico, solidão, isolamento social, afastamento das pessoas do seu cotidiano, vergonha, constrangimento devido à idade e a contaminação; 0 desespero toma conta com o medo das pessoas estarem cientes do diagnóstico.

Sendo assim, é notório que, mesmo possuindo conhecimento do que é necessário para prevenção e possuir comportamento sexual adequado visando a saúde sexual e proteção parcial entre a terceira idade, a concepção de morte que existe entre as pessoas quando é falado sobre HIV/AIDS se entrelaça com o pensamento estigmatizado que essas são doenças incuráveis e graves, que trazem medo do diagnóstico e consequentemente medo da morte (SANTOS MC, et al., 2020).

\section{Educação em saúde na atenção básica acerca da sexualidade das pessoas idosas}

A pesquisa realizada por Theis LC e Gouvêa DL (2019) pontuou em seus resultados que, os idosos continuam com impulso e atividade sexual, apesar das alterações fisiológicas, socioculturais e das suas 
comorbidades, praticam o sexo, porém com a frequência diminuída como gesto de carinho e respeitos aos seus cônjuges. O envelhecimento revela uma vida com modos de agir, pensar em expressar-se específico da etapa, devem ser respeitados e considerados. É interessante compreender que a sexualidade vai além da relação sexual, ela se caracteriza como algo interessante e sadio para todas as faixas etárias quando realizadas de modo Seguro, visando a saúde sexual dos envolvidos (LIMA JS, et al., 2021).

Desta forma, é de extrema importância a realização de intervenções alertando os resultados negativos de práticas sexuais desprotegidas, essas informações são relevantes para que a população se previna das infecções, o resultado de um dos estudos apontou que todos os idosos negaram o uso do preservativo, dessa maneira estão vulneráveis quanto às infecções sexualmente transmissíveis (ANDRADE J, et al., 2017).

Segundo Bezerra LL, et al. (2017) a prática de enfermagem referente à prevenção de infecções sexualmente transmissíveis evoluiu ao longo dos anos adquirindo condutas relacionadas ao cuidado, de maneira que envolvem repercussões práticas e comportamentais relacionadas à sexualidade. É responsabilidade do enfermeiro, bem como da equipe multiprofissional da saúde a execução de avaliações abrangentes, educação em saúde, aconselhamento, acompanhamento, imunizações, realizações de testes, tratamentos, busca ativa de parceiros quanto à prevenção e tratamentos de IST 's e apoio para tomadas de decisões.

Foi observado que um número grande de idosos utilizam o Sistema Único de Saúde. Segundo dados do Elsi-Brasil $75,3 \%$ dos cidadãos dependem do SUS, dessa forma é fica claro que os profissionais da rede devem empenhar-se para a realização de acolhimento aos idosos, oferecendo-Ihes bom atendimento com foco na prevenção, promoção e proteção (AMARAL SV, et al., 2020).

De acordo com Andrade J, et al. (2017) foi realizada uma coleta em indivíduos de ambos os sexos, atendidos no serviço de saúde, esses tiveram sorologia positiva para HIV, sífilis ou hepatite B de modo que não foram diagnosticados na rotina dos serviços de saúde. Assim, isso caracteriza a ineficácia dos atendimentos ao indivíduo, desconsiderando as medidas de controle. $\mathrm{O}$ estudo também apontou que a sorologia em idosos foi solicitada somente na atenção secundária e terciária, mostrando que alguns profissionais da atenção básica excluem os idosos como sendo vulneráveis às IST/AIDS.

Logo, frente às dificuldades enfrentadas no processo de cuidado ao idoso que, por vezes, adentra à sexualidade como desnecessária ou inexistente, é importante atentar-se aos detalhes, a realização da anamnese nos pacientes com visão holística da sua saúde. Os enfermeiros precisam desenvolver habilidade quanto ao atendimento dos idosos na área sexual, é preciso considerar a sexualidade sendo fisiológica do ser humano e deve ser priorizada (VIEIRA KF, et al., 2016, ZANCO MR, et al., 2020).

O estudo de Santos FM, et al. (2020) apresenta que os profissionais de saúde estão negligenciando a saúde sexual dos idosos, são desmotivados e não atendem as expectativas quanto aos seus deveres relacionados à prevenção das IST's em idosos, isso se torna um desafio para a saúde.

Pesquisa realizada com profissionais da área da saúde envolvidos no acompanhamento de idosos com diagnóstico de IST, aborda que há uma saturação psicológica intensa sobre esse grupo de profissionais devido ao envolvimento com os pacientes que possuem medo, insegurança, frustração, sofrimento, pessoas que vivenciam o preconceito e estigmas relacionados a IST, essa realidade desafia os profissionais a refletir sobre suas ações (CASSÉTTE JB, et al., 2016).

Os profissionais de saúde não estão habituados quanto à sexualidade da pessoa idosa, possuindo dificuldades para prestar atendimento. Devido a essa brecha na saúde, é considerada necessária a conscientização dos enfermeiros e a equipe de saúde quando a introdução dos idosos como foco nas estratégias ao combate a IST'S, logo que a vitalidade desse grupo vem aumentando (VIEIRA KF, et al., 2016, ZANCO MR, et al., 2020).

Assim, o estudo de Andrade J, et al. (2017) expõe que os idosos que apresentam história de IST possuíam 5 (cinco) vezes mais chances de contaminação, de outra forma, as mulheres possuíam 12 vezes mais chances para se contaminarem independente da história, a probabilidade é gigantesca frente aos homens idosos. 
O número de casos emergindo, tem sido um desafio para o Brasil, tendo como extrema necessidade a implementação de políticas públicas e estratégias de modo que haja um maior número de prevenção, melhor qualidade de vida e saúde para essas pessoas. As práticas de enfermagem quanto às IST'S está para além da execução de ações em educação e saúde, na sua totalidade precisa compor um cuidado completo de aconselhamento, notificação, encaminhamento, escuta qualificada, entre outros, incluindo exames que foram feitos pelos usuários da unidade, é importando a convocação do parceiro para acompanhamento, tratamento e aconselhamento conjunto (ALVES MA, et al., 2017).

Ressalta-se que, desenvolver e programar práticas de educação em saúde para idosos com foco na sexualidade é eficaz para construir um meio de prevenção e promoção à saúde, logo, os profissionais de saúde com base em seus conhecimentos possuem autonomia para aconselhar, criar, reforçar hábitos e condutas que favorecem as práticas de prevenção (THEIS LC e GOUVÊA DL, 2019).

Assim, os profissionais apontam que a sobrecarga do trabalho provoca efeitos desconstrutivos quanto ao atendimento aos idosos. Porém, são interessantes ações em educação em saúde voltadas para a prevenção e uso seguro de medicamentos, a criação de ideias, estratégias para trazer aos idosos assuntos que retratam a sexualidade sem ignorância, reforçar a paciência com a pessoa idosa quanto às dúvidas e esclarecer assuntos acerca da saúde sexual, quanto aos profissionais de saúde existe uma necessidade de aprimorarem seus conhecimentos com relação a HIV/AIDS (CASSÉTTE JB, et al., 2016)

Para alcançar o público das pessoas idosas os enfermeiros necessitam utilizar uma linguagem lúdica, inteligível, compreensível, evidenciando que o diálogo é uma boa estratégia para discussões relacionadas à sexualidade. Corroborando as ações educativas, e a criação de vínculo adentrando nas orientações auxilia na realização das práticas preventivas e consequentemente reduzem os números de casos de IST (RODRIGUES MS, et al., 2019).

\section{CONSIDERAÇÕES FINAIS}

Em síntese, através do estudo pode-se afirmar que fragmentos dos idosos possuem vida sexual ativa, uma vez que estão preservados os desejos sexuais, ainda que com as condições fisiológicas alteradas. No entanto, alguns idosos não compreendem que são vulneráveis a IST 's, tornando-se um problema devido à ausência de medidas de prevenção, e negligência em práticas sexuais seguras. Importante salientar que a ignorância, o preconceito, favorecem com que as políticas de prevenção a infecções sexualmente transmissíveis não estejam voltadas à pessoa idosa. Torna-se necessário a atuação dos profissionais de saúde quanto ao atendimento à pessoa idosa visando todos os aspectos da saúde proporcionando qualidade de vida e bem-estar sexual dos mesmos.

\section{REFERÊNCIAS}

1. ALVES MA, et al. As dificuldades enfrentadas pelo paciente idoso diagnosticado com o HIV: olhar do enfermeiro diante da problemática. Revista Saúde em Foco, 2017; 9: 692-700.

2. AMARAL SV, et al. Conhecimento e comportamento de um grupo de idosos frente às infecções sexualmente transmissíveis. Revista Eletrônica Acervo Saúde, 2020; 12(9): e3891-3891.

3. ANDRADE J, et al. Vulnerabilidade de idosos a infecções sexualmente transmissíveis. Acta Paulista de Enfermagem, 2017; 30(1): 8-15.

4. ARAÚJO JI, ANDRADE FG. Prolongamento da vida sexual e a população idosa: conquistas e desafios. Ciência da Saúde, 2019; 8: 16-26.

5. BEZERRA LL, et al. Abordagem das IST por enfermeiro (às): revisão integrativa de literatura. Anais II CONBRACIS... Campina Grande: Realize Editora, 2017.

6. BRITO NM, et al. Idosos, infecções sexualmente transmissíveis e AIDS: conhecimentos e percepção de risco. ABCS Health sci, 2016; 140-145.

7. CASSÉTTE JB, et al. HIV/aids em idosos: estigmas, trabalho e formação em saúde. Revista Brasileira de Geriatria e Gerontologia, 2016; 19(5): 733-744.

8. FERREIRA CO, et al. Vulnerabilidade a infecções sexualmente transmissíveis em idosos usuários de um centro de testagem e aconselhamento. Arquivos de Ciências da Saúde da UNIPAR, 2019; 23(3). 
9. SANTOS MC, et al. Diagnósticos de enfermagem para mulheres idosas com vulnerabilidade ao HIV/aids. Revista Brasileira de Enfermagem, 2018; 71.

10. INSTITUTO BRASILEIRO DE GEOGRAFIA E ESTATÍSTICA (IBGE). Estatísticas de população social, 2021. Disponível em: https://www.ibge.gov.br/estatisticas/sociais/populacao. Acessado em: 16, MAIO de 2021;

11. JÚNIOR PSS, MENDES PN. Sexualidade do idoso: instrumentos do enfermeiro para a prevenção das infecções sexualmente transmissíveis. Pesquisa, Sociedade e Desenvolvimento,2020; 9(12): 27491210760-27491210760.

12. LIMA JS, et al. O conhecimento dos idosos acerca das infecções sexualmente transmissíveis. Caderno de GraduaçãoCiências Biológicas e da Saúde-UNIT-ALAGOAS, 2021; 6(3): 31-31.

13. MONTE CF, et al. Idosos frente a infecções sexualmente transmissíveis: uma revisão integrativa. Brazilian Journal of Health Review, 2021; 4 (3): 10804-10814.

14. PINTO VM, et al. Fatores associados às infecções sexualmente transmissíveis: inquérito populacional no município de São Paulo, Brasil. Ciência \& Saúde Coletiva, 2018; 23: 2423-2432.

15. RODRIGUES MS, et al. Obstáculos enfrentados pela Enfermagem na prevenção de infecções sexualmente transmissíveis na terceira idade. Revista Eletrônica Acervo Saúde, 2019; 29: e1116-e1116.

16. SANTOS, FM et al. Idoso e hiv: um desafio para o enfermeiro nas estratégias de prevenção. BIUS-Boletim Informativo Unimotrisaúde em Sociogerontologia, 2020; 15(9): 1-10.

17. SANTOS MC, et al. Percepções e vivências de idosos sobre sua sexualidade. Almanaque multidisciplinar de pesquisa, 2017; 4(1).

18. SILVA AG, et al. Revisão integrativa da literatura: assistência de enfermagem à pessoa idosa com HIV. Rev Bras Enferm, 2018; 71(2): 939-47.

19. THEIS LC, GOUVÊA DL. Percepção dos Idosos em Relação a Vida Sexual e as Infecções Sexualmente Transmissíveis na Terceira Idade. Rev. bras. ciênc. saúde, 2019; 197-204.

20. VIEIRA KF, et al. A sexualidade na velhice: representações sociais de idosos frequentadores de um grupo de convivência. Psicologia: ciência e profissão, 2016; 36: 196-209.

21. ZANCO MR, et al. Sexualidade da pessoa idosa: principais desafios para a atuação do enfermeiro na atenção primária em saúde. Brazilian Journal of Health Review, 2020; 3(3): 6779-6796. 